\title{
Diferenciais de desempenho acadêmico dos alunos da UNILAB: uma análise sobre
}

\section{regressões quantílicas}

\author{
UNILAB students' academic performance differentials: an analysis on quantill regressions \\ Diferenciales de desempeño académico de estudiantes de UNILAB: un análisis sobre regresiones
} cuantiles

Recebido: 26/11/2021 | Revisado: 02/12/2021 | Aceito: 03/12/2021 | Publicado: 01/01/2022

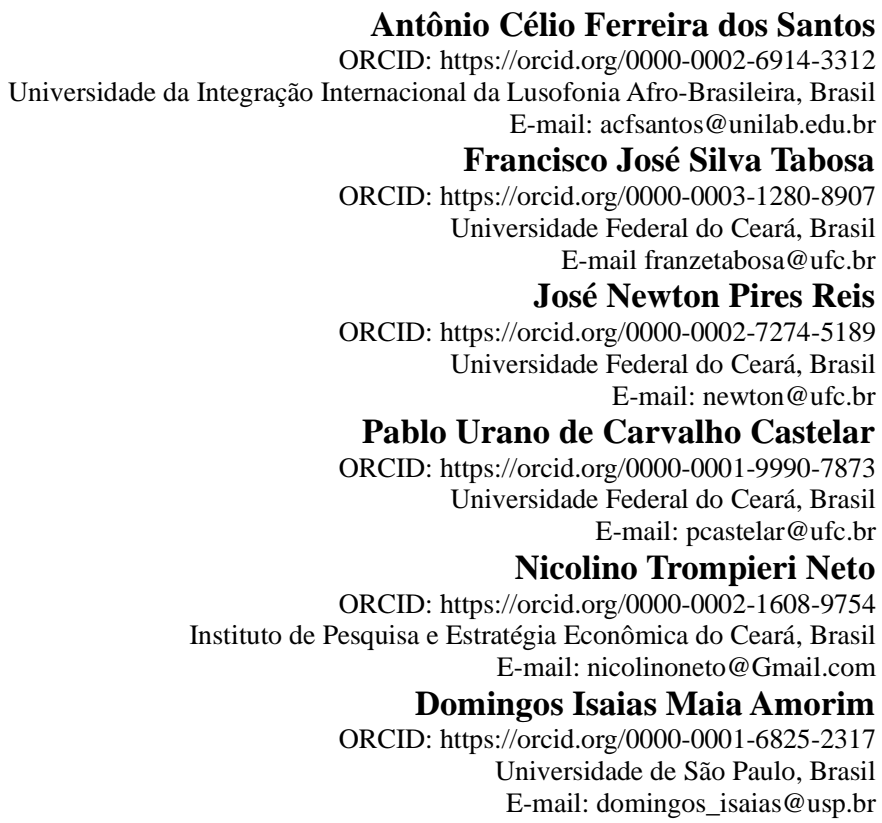

\section{Resumo}

Este trabalho tem como objetivo detectar efeitos nos quantis da distribuição do índice de desenvolvimento educacional (IDE - Índice de Desenvolvimento Educacional) para o caso da Universidade da Integração Internacional da Lusofonia Afro-Brasileira (UNILAB), permitindo identificar e verificar se determinado fator tem maior efeito em estudantes de alto, médio ou baixo desempenho acadêmico. Utilizou-se, para esse propósito, a metodologia de Regressões Quantílicas, com dados dos alunos da UNILAB para o semestre 2018.1. Dentre os principais resultados, constatou-se que a modalidade presencial tem um efeito positivo no IDE, tal que os melhores desempenhos são dos alunos com maior IDE; além disso, melhor desempenho também foi observado no caso das mulheres. Por outro lado, o fato de residir no município de Redenção, onde fica a Universidade, teve como resultado o inverso do esperado, ou seja, morar na cidade parecer ter impacto negativo no rendimento do discente.

Palavras-chave: Regressão quantílica; Desempenho acadêmico; UNILAB.

\begin{abstract}
This work aims to detect effects on the quantiles of the distribution of the educational development index (IDE Educational Development Index) for the case of the Universidade da Integração Internacional da Lusofonia AfroBrasileira (UNILAB), allowing to identify and verify whether a given factor has greater effect on students with high, medium or low academic performance. For this purpose, the Quantile Regression methodology was used, with data from UNILAB students for the 2018.1 semester. Among the main results, it was found that the in person classes modality has a positive effect on the IDE, such that the best performances are from students with the highest IDE; in addition, better performance was also observed in the case of women. On the other hand, the fact of living in the municipality of Redenção, where the University is located, had the opposite of what was expected, that is, living in the city seems to have a negative impact on the student's income.
\end{abstract}

Keywords: Quantile regression; Academic achievement; UNILAB. 


\section{Resumen}

Este trabajo tiene como objetivo detectar efectos sobre los cuantiles de la distribución del índice de desarrollo educativo (IDE - Educational Development Index) para el caso de la Universidad de Integración Internacional de la Lusofonia Afrobrasileña (UNILAB), permitiendo identificar y verificar si un determinado factor tiene mayor efecto en estudiantes con rendimiento académico alto, medio o bajo. Para ello se utilizó la metodología de Regresión Cuantil, con datos de estudiantes de UNILAB para el semestre 2018.1. Entre los principales resultados, se encontró que la modalidad presencia tiene un efecto positivo en la IED, de manera que los mejores desempeños son de los estudiantes con mayor IED; además, también se observó un mejor desempeño en el caso de las mujeres. Por otro lado, el hecho de vivir en el municipio de Redenção, donde se ubica la Universidad, tuvo lo contrario de lo esperado, es decir, vivir en la ciudad parece tener un impacto negativo en los ingresos de los estudiantes.

Palabras clave: Regresión cuantílica; Logro académico; UNILAB.

\section{Introdução}

Ao longo das últimas décadas no Brasil, nota-se que a avaliação em larga escala vem se consolidando como um instrumento das políticas públicas em educação, em seus diferentes âmbitos de gestão, seja em nível federal, estadual ou municipal. No entanto, ainda se observam inúmeros desafios quanto ao uso efetivo dessas avaliações na formulação, reformulação e no monitoramento de ações, projetos e programas educacionais, e, notadamente, no que diz respeito ao trabalho no interior das unidades escolares (Gimenes, 2015).

Além de serem avaliados oficialmente pelos órgãos federais, dentre os quais se destaca a avaliação dos alunos no ENADE (Exame Nacional de Desempenho de Estudantes), as IFES (Instituições Federais de Ensino Superior) criam suas próprias formas para avaliar seus discentes a partir de critérios pré-estabelecidos e aprovados em seus conselhos universitários. Estas formas de avaliação podem ajudar a entender os diferenciais de desempenhos entre os alunos e, consequentemente, podem ser utilizadas para a formulação de políticas públicas (pelos governos) e na gestão interna das universidades com a finalidade de melhorar a eficiência em seus resultados.

Nos estudos acerca dos fatores associados ao desempenho escolar, seja nos níveis fundamental, médio ou superior, tem destaque a metodologia de modelos hierárquicos, uma vez que existem fatores conjuntamente associados ao estudante e à escola onde estuda, ou seja, há um nível de hierarquia primário - os estudantes -, e um nível agregado, nomeadamente as escolas (Alves \& Soares, 2008; Nascimento, 2003; Pontes \& Soares, 2016).

Tendo em vista que a maioria das formas de analisar o desempenho de um estudante numa avaliação de larga escala é, geralmente, medida por uma escala contínua, a maioria dos estudos usa os denominados modelos hierárquicos lineares. Os modelos lineares hierárquicos podem ser considerados extensão da regressão linear múltipla para acomodar uma estrutura hierárquica. Por intermédio do modelo adotado nesse estudo, procura-se avaliar a parcela do desempenho devida a cada fator associado ao estudante, nomeadamente a modalidade de ensino (presencial ou à distância), renda familiar, tamanho da família, raça, sexo, local de residência e nacionalidade).

Tradicionalmente, os modelos relacionam o valor médio do desempenho dos estudantes com os diversos fatores extra e intraescolares. Mas muitos desses fatores podem de maneira diferente afetar estudantes que apresentam baixo e alto desempenhos. Notadamente, nas políticas educacionais que buscam melhorar o desempenho dos alunos, há interesse em encontrar fatores intraescolares com maior efeito em alunos que registram baixo desempenho.

Neste estudo, procura-se analisar o caso específico da Universidade da Integração da Lusofonia Afro-Brasileira (UNILAB), em Redenção, no Ceará, que tem por características, desde sua criação, levar a bom termo a implementação de políticas públicas direcionadas a questão racial (e a consequente política afirmativa), internacionalização, e atender as demandas de interiorização do ensino superior.

A partir de informações relativas aos estudantes da UNILAB, busca-se verificar quais fatores afetam o desempenho acadêmico desses universitários, recorrendo-se aos seus resultados registrados pelo IDE disponibilizados institucionalmente 
pelas Diretoria de Tecnologia da informação e o Centro de Processamento de Dados, responsável pelo Sistema Acadêmico (SIAC) da Instituição.

Dado o exposto, avalia-se o efeito dos diferentes fatores associados não só na média do desempenho dos estudantes da UNILAB (medidos pelo IDE), mas também em diversos quantis da distribuição, por meio de Regressões Quantílicas, permitindo assim verificar se determinado fator tem maior efeito para o correspondente desempenho (alto, médio e baixo) do estudante.

A UNILAB é um caso relevante para estudo, pois desde a sua criação, conforme se pode verificar no PDI da própria universidade, seu programa da assistência estudantil tem buscado ampliar e sedimentar suas ações afirmativas, aqui entendidas como diretrizes, dispositivos normativos, medidas, iniciativas, atividades que procurem compensar déficits culturais, formativos e psicossociais que representem perdas históricas cumulativas, de grupos sociais e culturais alvo de preconceitos e discriminações devidas aos fatores de sexo, gênero, etnia e raça.

Para além disso, vale ressaltar que, de acordo com a sua Lei de criação (Lei № 12.289 de 20 de julho de 2010), a Instituição tem como objetivo ministrar ensino superior, desenvolver pesquisas nas diversas áreas de conhecimento e promover a extensão universitária, e tendo como missão institucional específica formar profissionais e cidadãos para contribuir com a integração entre o Brasil e os demais estados-membros da Comunidade dos Países de Língua Portuguesa (CPLP), especialmente os países africanos (Angola, Cabo Verde, Guiné-Bissau, Moçambique, Portugal, São Tomé e Príncipe) e Timor Leste, bem como promover o desenvolvimento regional e o intercâmbio cultural, científico e educacional.

Esta pesquisa, além desta introdução, é composta por mais quatro seções. Na seção seguinte é feita breve revisão da literatura sobre avaliação de desempenho acadêmico, na qual está especificada a evolução da experiência brasileira. A metodologia utilizada está na terceira seção, na qual consta a estatística descritiva e o modelo proposto (regressões quantílicas). Os resultados e as discussões estão na quarta seção, seguidas, por fim, pelas considerações finais do estudo.

\section{Revisão de Literatura}

A avaliação é uma tarefa didática necessária e permanente do trabalho docente, que deve acompanhar passo a passo o processo de ensino e aprendizagem. Através dela, os resultados obtidos no decorrer do trabalho conjunto do professor e dos alunos são comparados com os objetivos propostos a fim de constatar progressos, dificuldades, e reorientar o trabalho para as correções necessárias (Libâneo, 1994).

Espera-se que, pelo ato de avaliar, possa ser analisada a qualidade do desempenho dos alunos. A literatura internacional costuma elencar fatores determinantes ao desempenho acadêmico. A título de ilustração, os fatores familiares e as características pessoais - idade, renda domiciliar, escolaridade dos pais, sexo, raça, entre outros constituem um desses determinantes. Questões relacionadas à qualidade do Ensino Médio são bastantes evidenciadas pela literatura internacional, a saber: recursos da escola, qualificação dos professores, passando pelo próprio desempenho do aluno nessa época que antecede a sua entrada na Universidade. Ou seja, o exame de entrada, comumente conhecido no Brasil como vestibular, também surge como fator explicativo à performance acadêmica dos alunos universitários (Farias, 2013).

Autores como Furtado (1954), Holanda (1956) e Freire (1951) já traziam o debate sobre o tema da qualidade da educação brasileira. Porém, o tema ocupava espaço marginal nas obras mais gerais acerca do pensamento social brasileiro. Vale ressaltar que, mais recentemente, diversos trabalhos que analisavam a qualidade levavam em consideração os recursos investidos na área.

O processo de avaliação do Ensino Superior no Brasil é recente: remonta ao início da década de 1960, coincidindo com o primeiro boom da educação superior no país (Zainko, 2008). Em 1983, foi criado o Programa de Avaliação da Reforma 
Universitária (PARU), que vigorou até 1984 e que tinha como intuito gerar dados para fomentar estudos e discussões sobre tal questão. Em razão do PARU, observou-se a ausência de parâmetros necessários para a avaliação de desempenho, a qual, por sua vez, contribuiu para determinar de forma efetiva alocação dos recursos públicos no setor.

Na década de 1990, o governo brasileiro passou a enfatizar os processos de avaliação de ensino em bases que visasse a qualidade. Em 1993, no governo Itamar Franco, foi criada pela Secretaria da Educação Superior a Comissão Nacional de Avaliação, cuja responsabilidade repousava na implantação de um programa de avaliação institucional nas universidades brasileiras (PAUB). Assim, o MEC passava a articular, viabilizar e financiar a avaliação do ensino superior (Zainko, 2008).

Pela Lei $n^{\circ} .9 .131$, de 24 de novembro de 1995, cria-se o Exame Nacional de Cursos, popularmente conhecido por Provão, numa tentativa pioneira de objetivamente avaliar a performance das IES e detectar a aptidão dos estudantes em fase de conclusão dos seus respectivos cursos de graduação.

Não obstante, a partir da Lei no 10.861, de 14 de abril de 2004, criou-se o Sistema Nacional de Avaliação da Educação Superior (SINAES) que, dentre seus instrumentos para medir a qualidade da educação superior, conta com o Exame Nacional de Desempenho de Estudantes - ENADE (INEP, 2013). A partir dessa data, o ENADE substituiu o Provão.

Nos últimos anos, com mais frequência tem sido a discussão acerca da natureza do desempenho educacional no Brasil. Em geral, tem-se enfatizado apenas alguns determinantes em detrimento de outros como evidencia a literatura empírica nesta área. Por exemplo, segundo Barros e Mendonça (1996), o ambiente familiar tem seguramente impacto sobre o desempenho educacional dos estudantes brasileiros. Fatores como este são frequentemente abordados pelos autores que constituem a literatura internacional acerca do tema.

Com efeito, apesar da melhoria educacional verificada no Brasil nos últimos 20 anos, a evolução da educação foi mais lenta do que a dos demais países da América Latina (Menezes-Filho, 2001). De fato, mesmo com a ampliação, nos últimos anos, do acesso à educação básica, no Brasil, para grande parte das classes de renda mais baixa, a demanda pelo ensino superior cresceu intensamente.

Sendo assim, apesar do considerável crescimento das vagas universitárias, os jovens pobres e negros seguem com baixa representação entre os ingressantes na universidade pública - em geral, a mais concorrida (Menezes-Filho et al., 2019).

Atualmente, a avaliação é um dos pilares da política educacional do Ministério da Educação (MEC). As três principais inovações inseridas no Plano de Desenvolvimento da Educação (PDE), criado em 2007 pelo MEC, foram: i) incorporação dos objetivos de accountability; ii) criação de um indicador sintético da qualidade da educação básica, que considera tanto o desempenho dos estudantes em exames padronizados quanto a progressão desses alunos no sistema; e iii) definição de metas seja para o país, seja para cada sistema e escola em particular (Fernandes \& Gremaud, 2009).

Amaral e Menezes-Filho (2008) estudaram a relação entre os gastos educacionais e o desempenho escolar no Brasil, e neste trabalho, fica clara a grande dispersão existente nos gastos municipais no ensino fundamental: enquanto a média dos gastos é de $\mathrm{R} \$ 1.015,34$, seu desvio-padrão é de $\mathrm{R} \$ 1.165,42$. Além disso, o artigo atenta para o fato segundo o qual a relação entre gastos educacionais e qualidade do ensino é significante apenas para os municípios com notas mais altas na Prova Brasil.

Do ponto de vista geral, o governo brasileiro intensificou, de fato, a avaliação em larga escala a partir do início da década de 1990 e encontrou contexto particularmente fértil para sua consolidação a partir de 1995 (Bonamino, 2002). Seguindo os desenvolvimentos já em curso, em 1996 a LDB explicitou que “compete à União assegurar o processo nacional de avaliação do rendimento escolar no ensino fundamental, médio e superior, em colaboração com os sistemas de ensino, objetivando a definição de prioridades e a melhoria do ensino".

Outro aspecto que deve ser levado em consideração ao se analisar os desempenhos doa alunos, apontados por diversos autores, é a renda, tomada como fator determinante, ou seja, quanto maior a renda melhor o desempenho dos alunos. Como exemplo, pode ser citado o estudo longitudinal, com a base de dados do Saeb, elaborado por Leon e Menezes- Filho (2003), em 
que consta a classificação dos níveis socioeconômicos em quantis e o efeito da seleção na progressão escolar a partir dos níveis de renda. As simulações mostraram que a renda se tornou mais determinante na probabilidade de reprovação ao longo do período, revelando que, para a $8^{\mathrm{a}}$ série e o $3^{\circ}$ ano do ensino médio, os estudantes com maior renda apresentam menor chance de reprovação sob os efeitos marginais de 1996-1997, do que se deparassem com os efeitos marginais de 1984-1985.

Um trabalho que utiliza metodologia similar à adotada no presente trabalho é o de Barbetta, et al., (2018), que realizam um estudo de fatores associados ao desempenho escolar usando regressão quantílica, e em especial regressão quantílica hierárquica. A metodologia proposta é aplicada no sistema de avaliação em larga escala do estado do Pará (SisPAE), na sua edição de 2016, que avalia os estudantes nas áreas de Língua Portuguesa e Matemática. Os autores encontram que o nível socioeconômico em que o estudante está inserido provoca maiores diferenças em estudantes de alto desempenho, o que também ocorre com o efeito positivo de o professor se preocupar com os deveres de casa. Estudantes com desempenho baix o parecem mais dependentes do professor, pois, de acordo com os autores, a concordância de que o aprendizado depende mais do professor do que do aluno tem alto efeito negativo nos vários quantis da distribuição. Com a abordagem da regressão quantílica, verificou-se que esse efeito negativo é mais forte nos grupos de desempenho mais alto, ou seja, estudantes com bom desempenho que concordam com essa afirmação tendem a ficar bem abaixo daqueles com bom desempenho que discordam.

Outro estudo relevante no contexto brasileiro foi realizado por Arraes \& Mariano (2019), que busca evidências sobre os diferenciais de desempenho entre os alunos de nível médio de escolas privadas regulares e escolas públicas. As escolas públicas, na amostra, representadas pelas profissionalizantes que detêm os alunos com melhores resultados escolares. A aplicação empírica diferencia-se pela utilização de regressão quantílica incondicional (RQI) com aplicação da decomposição de Oaxaca-Blinder. Os resultados da pesquisa sugerem haver superioridade dos alunos das escolas privadas em todas as áreas do Exame Nacional do Ensino Médio (Enem), principalmente em redação, cujos diferenciais de performance oscilaram entre $12 \%$ e $16 \%$ ao longo da distribuição. A decomposição detalhada dos efeitos indicou, ainda, que escolaridade e renda dos pais se destacam para explicar tais diferenciais

Já Mendes, et al., (2020) analisam o papel do acesso à informação no âmbito da educação superior, controlando para fatores como escolaridade do pai, sexo, cor e esforço individual, com base nas informações acadêmicas e socioeconômicas de uma amostra de estudantes da Universidade Federal da Paraíba (UFPB), entre 2001 e 2010. A estratégia empírica consistiu em estimações por mínimos quadrados ordinários no intuito de analisar o impacto médio das variáveis sobre o coeficiente de rendimento acadêmico, assim como utiliza regressão quantílica, tendo como propósito contornar os problemas de heterogeneidade dos indivíduos. Os resultados indicam que o acesso à informação impacta positivamente as notas dos discentes e diminui sua importância ao longo da distribuição condicional do coeficiente de rendimento acadêmico. Além disso, aqueles alunos que têm pais com, no mínimo, ensino superior, assim como aqueles que são brancos e do sexo masculino, apresentam melhor desempenho em relação aos demais, com maior ênfase nos que possuem menores notas. Por fim, o coeficiente de rendimento acadêmico parece não depender de esforço individual.

Vale destacar que no Brasil, as universidades criam suas próprias formas de avaliação de seus discentes com base em critérios pré-estabelecidos e aprovados em seus conselhos universitários, e que nos últimos anos o Brasil vem democratizando o acesso ao ensino superior, objetivando amenizar a dívida histórica com os afrodescendentes e diminuir o hiato existente entre alunos que concluem o ensino médio nas redes públicas e privadas.

Neste presente estudo, a qualidade a ser analisada corresponde ao desenvolvimento dos alunos da UNILAB medidos por um índice cuja fórmula parte de uma definição interna da própria universidade, o IDE - Índice de Desenvolvimento do Estudante.

De fato, conhecer os principais fatores que afetam o desempenho estudantil auxilia os gestores e os professores a orientarem suas ações de forma mais objetiva e pragmática, com a consequente melhoria no desempenho acadêmico e na 
diminuição das diferenças entre eles.

\section{Metodologia}

Quanto ao tipo de pesquisa e com fundamentos em Gil (2008) e Severino (2013) este artigo se caracteriza como uma pesquisa aplicada tendo por finalidade entender o diferencial de desempenho dos alunos da UNILAB. Quantos aos objetivos, a pesquisa se caracteriza como uma pesquisa exploratória e descritiva, pois busca ampliar o conhecimento de determinado assunto ao mesmo tempo que estuda as suas características e seus problemas (Severino, 2013). Quanto a abordagem, constituise de uma pesquisa quantitativa, pois utiliza de instrumentos estatísticos e econométricos, tanto na coleta como no tratamento dos dados (Gil, 2008; Severino, 2013).

\subsection{Bases de dados}

A metodologia proposta é aplicada no sistema de avaliação do desempenho do discente dos alunos da graduação da UNILAB, o qual é calculado pelo Índice de Desempenho do estudante (IDE). A avaliação envolveu uma concernente a estudantes ativos nos cursos de graduação presencial e à distância da UNILAB, no ano de 2018 que fizeram matrícula no semestre 2018.1. A escolha do semestre 2018.1 se deu por ser o período de consolidação de uma base de dados mais sólida e confiável feita a partir de estudos, aperfeiçoamento de coletas de dados e discussões internas na universidade, e que proporcionaram a partir de então, obter informações de variáveis importantes para as análises dos modelos propostos nesta pesquisa.

Para cada elemento da amostra, existem informações socioeconômicas declaradas durante a realização da inscrição no processo seletivo e algumas de quando ingressado na universidade. Os dados foram coletados junto à DTI (Diretoria de Tecnologia da informação), no Sistema Acadêmico (SIAC) e pelo Centro de Processamento de Dados. Os dados obtidos apresentam, além das habituais informações socioeconômicas dos ingressantes, informações sobre número de matrícula e acompanhamento acadêmico do estudante no decorrer do curso. A amostra abrange 2967 observações, entre alunos que ingressaram por meio do sistema de reserva de vagas, vestibular de ampla concorrência e processos específicos para alunos estrangeiros e EAD.

As variáveis socioeconômicas utilizadas na análise, com exceção das variáveis renda familiar, IDE e tamanho da família, as demais variáveis são dicotômicas, com valor igual à unidade, indicando que aquele aspecto pode ser atribuído ao indivíduo, caso contrário, atribui-se o valor zero.

A UNILAB acompanha o desempenho de seus alunos baseado numa avaliação de aprendizagem, aprovada no Conselho universitário na resolução 27 de 11 de novembro de 2014. Esta avaliação foi constituída como:

- Parte integrante do ensino aprendizagem;

- Processo que subsidia a reflexão e prática docente;

- Elemento constitutivo do processo formativo do estudante.

A avaliação de aprendizagem na UNILAB visa o êxito acadêmico do discente de graduação calculado por um componente curricular, em que se combina o aproveitamento acadêmico e a assiduidade. $\mathrm{O}$ aproveitamento acadêmico consiste no êxito discente de cada componente em cada disciplina e a assiduidade se refere a frequência regular às atividades acadêmicas de cada componente curricular.

O desempenho do discente da graduação é calculado pelo IDE, isto é, trata-se da média das notas finais das disciplinas ponderada pela carga horária respectiva, multiplicada por um redutor por reprovação (falta e/ou trancamento) de disciplina, conforme exposto abaixo: 
$\mathrm{IDE}=\left(\frac{\sum_{i=1}^{n} N O_{i} \cdot C H}{\sum_{i=1}^{n} C H_{i}}\right)\left(1-\frac{0,3 \cdot N T R+0,7 \cdot N R F}{N T}\right)$

Em que:

- IDE - Índice de Desempenho do estudante;

- $\quad i$ - $i$-ésima disciplina que o estudante concluiu, com ou sem reprovação (com exceção daquelas em que houve reprovação por falta);

- $\quad n$ - total de disciplinas concluídas, com ou sem reprovação (com exceção daquelas em que houve reprovação por falta);

- $\quad$ NTR - número de disciplinas trancadas;

- $\quad \mathrm{NRF}$ - número de disciplinas com reprovação por falta;

- $\quad \mathrm{NT}$ - número total de disciplinas que o aluno teve matrícula efetivada;

- $\mathrm{NO}_{\mathrm{i}}$ - nota em que o aluno obteve em uma dada disciplina $i$; e,

- $\quad \mathrm{CH}_{\mathrm{i}}$ - carga horária da disciplina $i$.

OBS1: O primeiro fator, $\left(\frac{\sum_{i=1}^{n} N O_{i} \cdot C H}{\sum_{i=1}^{n} C H_{i}}\right)$ representa a fórmula básica, que é a média ponderada das notas obtida nas disciplinas pela carga horária. Exclui-se desta fórmula as notas e carga horária da disciplina em que o estudante obteve reprovação por falta.

OBS2: O segundo fator, $\left(1-\frac{0,3-N T R+0,7-N R F}{N T}\right)$ é um fator de redução. Caso o estudante não tenha apresentado trancamentos ou reprovações por falta, a segunda parcela do fator de redução se anula e a fórmula é multiplicada por 1, não havendo qualquer perda para o estudante.

Estima-se o modelo a partir de variáveis socioeconômicas, as quais encontram-se elencadas no Quadro 1. Com exceção da variável renda familiar, tamanho da família e IDE, as demais variáveis são dicotômicas, com valor igual à unidade indicando que aquele aspecto pode ser atribuído ao indivíduo (caso contrário, atribui-se o valor zero).

Quadro 1 - Definição das variáveis do modelo de regressão quantílica.

\begin{tabular}{|l|l|}
\hline Variável & Definição \\
\hline Modalidade & Dummy 1 se presencial, 0 se a distância \\
Tamanho da família & Número de pessoas na família \\
Renda familiar & Rendimento mensal da família (R $\$)$ \\
Sexo & Dummy 1 se do sexo masculino, 0 se do sexo feminino \\
Estrangeiro & Dummy 1 se Estrangeiro, 0 se brasileiro \\
Reside em Redenção & Dummy 1 se reside em Redenção, 0 se não reside em Redenção \\
Cotista & Dummy 1 se for Cotista, 0 se não for Cotista \\
$I D E$ & Nota obtida em uma escala de 0 a 100 \\
\hline
\end{tabular}

Fonte: Autores.

Salienta-se que foi considerada a renda familiar, o valor monetário declarado pelos estudantes no preenchimento das informações quando da inscrição; e, neste estudo, foram retirados outliers, ao considerar a possibilidade de erros de 
preenchimento dos formulários e/ou valores muito discrepantes do restante da população.

\subsection{Regressões Quantílicas}

Regressão quantílica é uma técnica estatística baseada em uma generalização do conceito de regressão por MQO, recorrendo à estimação de vários quantis da distribuição condicional associada ao modelo (Koenker \& Hallok, 2000). Enquanto nas técnicas dos modelos de regressão usuais estimam o valor médio da distribuição condicional da variável dependente, na metodologia da regressão quantílica é possível estimar toda uma família de quantis, fornecendo informações mais completas sobre a relação existente entre a variável resposta e as variáveis explicativas do modelo.

Segundo Koenker \& Bassett (1978), a função quantílica condicional (FQC) no quantil $p$ para uma variável aleatória contínua $y_{i}$ com uma função densidade bem comportada, dado um vetor de regressores $X_{i}$, pode ser definida como:

$$
Q^{(p)}\left(y_{i} \mid X_{i}\right)=F^{-1}\left(p \mid X_{i}\right)
$$

Tem-se que a FQC da RQ é a versão quantílica condicional da função expectativa condicional (FEC), a qual é derivada da solução do problema de minimização do erro médio ao quadrado, base da regressão linear múltipla (RLM). Neste caso, a média é o valor que minimiza o erro médio ao quadrado. Para a regressão quantílica, tem-se que o quantil desejado minimiza a esperança dos desvios absolutos.

Com relação à regressão linear, os estimadores de mínimos quadrados dão os valores dos coeficientes da regressão, $\hat{\beta} s$, que minimizam a soma dos quadrados dos resíduos, da seguinte forma:

$$
\operatorname{mim} \sum_{i}\left(y_{i}-x^{\prime} \beta\right)^{2}
$$

Por outro lado, os coeficientes da $\mathrm{RQ},{ }^{\beta^{(p)} s}$, solucionam o seguinte problema de minimização:

$$
\operatorname{mim} \sum_{i}\left|y_{i}-x^{\prime} \beta\right|
$$

Detalhadamente, os ${ }^{(p)_{S}}$ estão envolvidos na minimização da seguinte expressão:

$$
\sum_{i=1}^{n}\left(d_{p}\left(y_{i}, \hat{y}_{i}\right)\right)=p \sum_{y_{i} \geq x i \beta^{(p)}}\left|y_{i}-x^{\prime} \beta^{(p)}\right|+(1-p) \sum_{y_{i}<x i \beta^{(p)}}\left|y_{i} x^{\prime} \beta^{(p)}\right|
$$

Em que $d p$ é distância do valor de $Y$ com relação à sua média estimada $\widehat{y}_{i}$, e $p$ e (1-p) são os pesos atribuídos aos desvios.

Se estes desvios fossem iguais ou superiores à $\widehat{y}_{i}$, receberão peso $p$, caso sejam inferior, receberão peso $(1-p)$. Logo, os valores $\hat{\beta}^{(p)} s$ são aqueles que minimizam a soma ponderada dos desvios absolutos entre $y_{i}$ e o valor ajustado $\hat{y}_{i}=x^{\prime} \hat{\beta}^{(p)}$ (SPDA).

Assim,

$$
\beta^{(p)}=\arg _{\mathrm{b}} \operatorname{mim} E\left|y_{i} x^{\prime} b^{(p)}\right|
$$


Como a função objetivo (05) não é diferenciável, há a necessidade de ser otimizada pelo método simplex, que promove interações entre os números, a fim de minimizar as somas dos desvios na expressão. Os estimadores $\hat{\beta}^{(p)^{t}} s$ que conduzem ao valor mínimo dessas somas possuem boas propriedades assintóticas. Sob condições gerais, eles são assintoticamente normais (Cameron \& Trivedi, 2009).

Segundo Fonseca \& Martins (1987), para avaliar o grau de afastamento de uma distribuição em relação à unidade de referência, deve-se usar medidas de assimetria; e se numa distribuição, a mediana, a moda e a média forem iguais, tem-se uma distribuição simétrica. Mas, se a mediana for maior do que a média e menor do que a moda, então a distribuição é assimétrica à esquerda (assimetria negativa). E, se a mediana for maior do que a moda e menor do que a média, tem-se assimetria à direita (distribuição assimétrica positiva).

\section{Resultados e Discussão}

Nessa seção, encontram-se os resultados das estatísticas descritivas, bem como a discussão dos resultados de regressões quantílicas hierárquicas aplicadas aos desempenhos dos alunos da UNILAB medidos pelo IDE. A obtenção das estimativas teve por base um conjunto de 2967 estudantes, que correspondem às unidades observacionais com respostas nas variáveis em estudo.

Para os dados da amostra, a Tabela 1 apresenta uma análise exploratória dos dados. Observa-se que 89,2\% dos alunos estudam presencialmente com uma renda média em torno de $\mathrm{R} \$ 1.205,00$ reais e quantidade média de membros da família de 4,69 membros. Constata-se, ainda, que a maioria dos alunos da amostra são mulheres, sendo que do total 47,6 são homens, 34,7 são negros.

Tabela 1. Estatística descritiva do modelo de regressão quantílica.

\begin{tabular}{c|cccccc}
\hline Variáveis & Média & Desvio Padrão & Min & Máx & CV \\
\hline Renda familiar & 1205,821 & 1273,289 & 100 & 20000 & 1,055 \\
Tamanho da família & 4,699 & 2,847 & 1 & 26 & 0,606 \\
Sexo & 0,476 & 0,499 & 0 & 1 & 1,047 \\
Estrangeiro & 0,209 & 0,407 & 0 & 1 & 1,942 \\
Reside em Redenção & 0,258 & 0,438 & 0 & 1 & 1,691 \\
Cotista & 0,178 & 0,382 & 0 & 1 & 2,146 \\
IDE & 76,267 & 14,329 & 2,58 & 98,42 & 0,188 \\
\hline
\end{tabular}

Fonte: Autores.

Tratando-se das regressões quantílicas, as quais destacam a quantidade nas diferenças de valores das variáveis explicativas - ou da presença de fatores que costumam afetar o desempenho - alteram os valores dos quantis e, mais ainda, se o efeito é maior (ou menor) nos quantis que separam os estudantes de desempenho mais baixo ou nos quantis que separam aqueles de desempenho mais alto.

Os resultados dos modelos de regressão quantílica foram estimados para os quantis $0,10,0,25,0,50,0,75$ e 0,90, para as variáveis observadas, as quais usou-se como base: modalidade (EAD); sexo (mulher); negro (não negro); cotista (não cotista); reside em Redenção (não residente em Redenção); estrangeiro (ser nativo).

Pela Tabela 2, as variáveis modalidade, renda familiar e reside em Redenção mostraram-se estatisticamente significantes ao nível de significância de $(0,10)$ para quase todos os quantis analisados, com exceção do quantil $\left(q_{50}\right)$ para a variável renda e $\left(q_{75}\right)$ para reside em Redenção. $\mathrm{O}$ fato segundo o qual o estudante estuda presencialmente impacta positivamente a nota dele no IDE, sendo que o maior impacto se relaciona a alunos com menores desempenhos ( $\mathrm{q}_{10}$ ), cujo 
aumento é da ordem de 33,86 pontos.

A partir deste resultado, pode-se evidenciar que os alunos que participam de aulas presenciais têm como consequências melhores resultados relativamente aos alunos de menor desempenho acadêmico na UNILAB. Ressalta-se ainda que o menor efeito positivamente foi nos alunos de melhor desempenho q90, cujo aumento foi de 8,6 pontos nas notas dos alunos. Estes fatos podem ser observados também na figura 1. (Mudança nos Coeficientes Quantílicos-Modalidade), os quais mantém um padrão negativamente inclinado na medida em que aumenta o quantil.

Em relação a renda familiar, tem-se efeitos destoantes, o qual se observa que para os alunos de menor desempenho ( $q_{10}$ e $\left.q_{25}\right)$ até a mediana $\left(q_{50}\right)$ o efeito foi negativo, enquanto para os alunos de melhor desempenho $\left(q_{75}, q_{90}\right)$, o efeito foi positivo, conforme também se pode verificar na figura 1. (Mudança nos Coeficientes Quantílicos - Renda).

Figura 1. Mudança nos Coeficientes Quantílicos
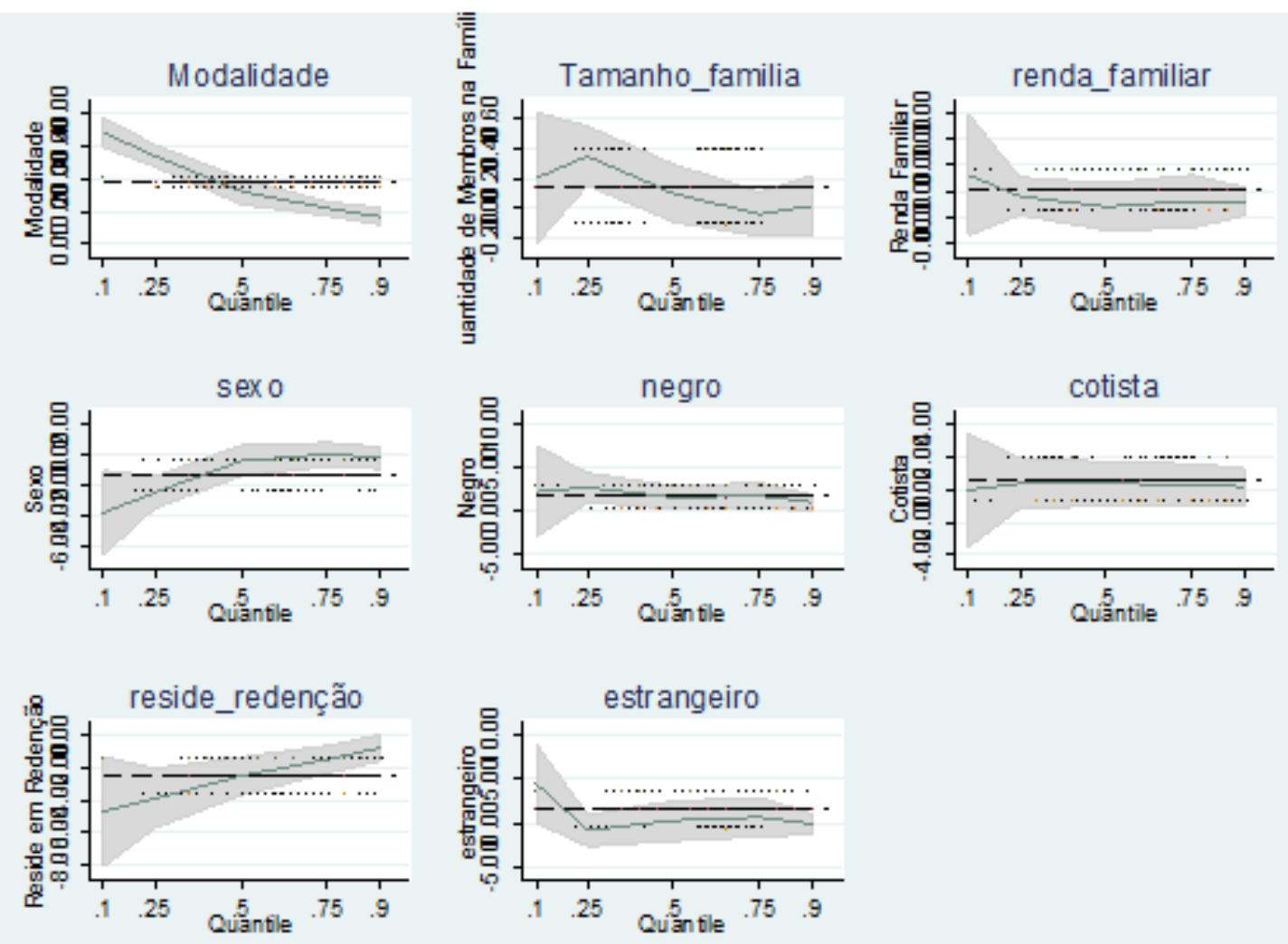

Fonte: Elaborado pelos autores.

Quando se analisam os resultados da Tabela 2, observados os coeficientes das variáveis do modelo de regressão quantílica, pode-se perceber algumas peculiaridades para os grupos de alunos divididos segundo os resultados do IDE por quantis.

O fato de o aluno residir em Redenção evidenciou para os alunos da UNILAB efeitos negativos, sendo que tais efeitos foram maiores nos alunos de menor desempenho $\left(q_{10}\right)$ e os de menores efeitos nos de maior desempenho. Para aqueles, a redução foi de $(4,7)$ pontos e para estes $(-0,7)$ ponto. Isto pode ser explicado pelo fato de parte considerável dos alunos estrangeiros moraem Redenção; e sabe-se que há uma deficiência ao entrar na universidade na língua portuguesa e na matemática, o que reduz, pelo menos no início, as notas do IDE.

Para a variável cotista, em que se analisa o aluno é ou não cotista, não se constata significância estatística para 
nenhum dos quantis considerados. Este fato pode ser explicado pela grande diferença entre o número de não cotistas comparativamente ao de cotistas, algo em torno de 4,5 vezes a mais. Quando se divide em grupos através de quantis, pode-se gerar resultados insignificantes. Já o sexo mostrou-se significante para os alunos de menor desempenho (q10 e q25), portanto, o fato de ser homem implica um efeito negativo no desempenho das notas que compõem o IDE. Todavia, observa-se que o efeito negativo é maior nos alunos de menor desempenho (q10), cuja redução na nota é de 3,8 pontos.

$\mathrm{O}$ fato de o aluno ser negro apresentou um efeito positivo nas notas do IDE para o grupo de alunos pertencentes a mediana e o quantil (q25), sendo que para a mediana, a nota relativa ao IDE é aumentada em 1,6 ponto e para o quantil $\left(q_{25}\right) 2,7$ pontos.

A variável estrangeiro é levada em consideração se os alunos vêm de outros países ou naturalizados no Brasil; e para os dados em análise, foi verificado que apenas o quantil ( $\left.\mathrm{q}_{10}\right)$, que ilustra os menores desempenhos, manifestou-se estatisticamente significante. $\mathrm{O}$ efeito apresentado foi positivo e para este grupo, com menores desempenhos, $\mathrm{o}$ fato de $\mathrm{o}$ aluno ser estrangeiro aumentou em 4,4 pontos a nota do IDE, o que sugere que caso seja analisado apenas o grupo de alunos de menor desempenho, os estrangeiros, para o caso específico da UNILAB, obtiveram melhores resultados.

Em relação ao tamanho da família, esta variável também se mostrou significante em apenas um quantil, no caso em questão, positivo o efeito para o quantil $\left(q_{25}\right)$, aumentando o resultado do IDE em 0,34 ponto. 
Tabela 2. Coeficientes das variáveis do modelo de regressão quantílica.

\begin{tabular}{|c|c|c|c|c|c|}
\hline \multirow[b]{2}{*}{ Variável } & \multicolumn{5}{|l|}{ QUANTIL } \\
\hline & $\mathrm{q}_{10}$ & $\mathrm{q}_{25}$ & $\mathrm{q}_{50}$ & $\mathrm{q}_{75}$ & $\mathrm{q}_{90}$ \\
\hline \multirow{2}{*}{ Constante } & $28,75^{*}$ & $45,79 *$ & $64,35^{*}$ & $74,79 *$ & $80,95^{*}$ \\
\hline & 3,43 & 2,186 & 2,198 & 1,168 & $-1,101$ \\
\hline \multirow{2}{*}{ Modalidade } & $33,86^{*}$ & $26,85^{*}$ & $16,10^{*}$ & $10,88^{*}$ & $8,628^{*}$ \\
\hline & 3,19 & 2,034 & 2,097 & 1,062 & (0.999) \\
\hline \multirow{2}{*}{ Tamanho da família } & 0,197 & $0,347^{*}$ & 0,099 & $-0,035$ & $1,80 \mathrm{e}-02$ \\
\hline & 0.291 & 0,158 & 0,12 & 0,0864 & $-8,30 \mathrm{e}-02$ \\
\hline \multirow{2}{*}{ Renda familiar } & $-8,21 \mathrm{e}-3 *$ & $-4,16 \mathrm{e}-3 *$ & $-2,20 \mathrm{E}-03$ & $3,17 \mathrm{e}-4^{*}$ & $3,1 \mathrm{e}-4 *$ \\
\hline & $-5 . e-3$ & $-2,34 \mathrm{e}-3$ & 2,144 & $2,29 \mathrm{e}-04$ & $-1,40 \mathrm{e}-03$ \\
\hline \multirow[t]{2}{*}{ Sexo } & $-3,839 *$ & $-2,474 *$ & $-0,435$ & $-0,0204$ & $-0,231$ \\
\hline & 1,38 & 0,739 & 0.469 & 0,406 & $-0,37$ \\
\hline \multirow[t]{2}{*}{ Negro } & 2,289 & $2,703^{*}$ & $1,601^{*}$ & 1,833 & 1.023 \\
\hline & 2,032 & 0,981 & 0,637 & 0,585 & $(0.439)$ \\
\hline \multirow{2}{*}{ Cotista } & $-0,046$ & 0,375 & 0,374 & 0,304 & 0,181 \\
\hline & 2,078 & 0,893 & 0,565 & 0,523 & $-0,545$ \\
\hline \multirow[t]{2}{*}{ Reside } & $-4,715^{*}$ & $-3,860^{*}$ & $-2,484^{*}$ & $-1,455$ & $-0,742^{*}$ \\
\hline & 1,559 & 0,845 & 0,596 & 0,462 & 0,343 \\
\hline \multirow[t]{2}{*}{ Estrangeiro } & $4,465^{*}$ & $-0,919$ & 0,196 & 0,599 & $-0,151$ \\
\hline & 2,521 & 1,386 & 1,179 & 0,832 & 0,690 \\
\hline Pseudo R2 & 0.172 & 0.125 & 0.073 & 0.049 & 0.037 \\
\hline
\end{tabular}

Nota: Usou-se como base: modalidade (EAD); sexo (mulher); negro (não negro); cotista (não cotista); reside em Redenção (não residente em Redenção); estrangeiro (ser nativo). Sendo: $\mathrm{P}<0,10^{*}$. Fonte: Autores.

Em suma, ao analisar os resultados, é importante considerar o contexto específico da UNILAB, com seu contingente significantes de alunos estrangeiros, oriundos de países com características próprias de contexto socioeconômico e, principalmente, ensino na educação anterior à universidade.

\section{Considerações Finais}

Neste trabalho, discutiu-se os fatores por trás do desempenho dos estudantes da UNILAB no semestre 2018.1. Utilizou-se os dados disponibilizados pela DTI, para estimar o modelo econométrico de regressões quantílicas para os 2.967 alunos avaliados naquele ano.

Nessa base de dados utilizada, constam características pessoais e socioeconômicas dos alunos. Observou-se que a variável referente à modalidade de estudo presencial se revelou estatisticamente significante em todos os quantis, visto que foram observados os efeitos positivos em cada quantil, sendo o maior o que diz respeito aos alunos com menor desempenho acadêmico.

Em decorrência disso, resta evidente que para estes alunos certamente é importante a presença física do professor, fato 
este que corrobora com outros estudos já feitos, onde se observa que alunos com melhor desempenho, são mais autodidatas e aproveitam melhores as tecnologias EAD.

Em relação à renda familiar, destaca-se uma discrepância em relação aos efeitos apresentados nos extremos dos quantis; e, em relação ao sexo para os alunos de menor desempenho o fato de o aluno ser homem reduziu a nota em -3,8 para o quantil $\left(q_{10}\right)$ e -2,4. Observa-se ser recorrente na literatura evidenciar que as mulheres obtêm, em média, melhores resultados que os homens.

Espera-se que quanto menor for a distância da casa dos estudantes para o seu local de ensino os resultados acadêmicos sejam mais robustos, dada a perda de horas de estudo com deslocamento. Entretanto, o resultado apresentado neste estudo contrariou isso. Uma provável explicação está associada ao fato da maioria dos alunos residentes em Redenção ser estrangeira. Por sua vez, estes discentes, historicamente, ingressam na universidade com certo déficit de aprendizagem, sobretudo na Língua Portuguesa e Matemática.

Dado este cenário, cursos de nivelamento têm sido criados pela UNILAB. Com o tempo, será possível analisar as diferenças de notas entre alunos que fizeram o curso de nivelamento e os que não fizeram ou não o concluíram.

Os resultados com respeito aos alunos da UNILAB vêm encorajar novos estudos, em outras populações, que possam corroborar várias relações discutidas neste trabalho. Sabe-se que há políticas públicas realizadas com o objetivo de atenuar as diferenças de desempenho entre os estudantes, na tentativa de melhorá-lo, especialmente, daqueles estudantes com maior dificuldade de aprendizado.

Para uma correta análise deste tipo de problemática, a regressão quantílica hierárquica torna-se um adequado instrumento na avaliação do efeito dessas políticas, pois mede o efeito dos fatores de interesse em diferentes níveis do desempenho educacional, não só em seu valor esperado.

Para trabalhos futuros fica como sugestão captar o efeito da Pandemia do Covid-19 no IDE dos alunos da UNILAB.

\section{Referências}

Acemoglu, D. \& Angrist, J. (2000) How large are the social returns to education? Evidence from compulsory schooling laws. NBER Working papers, 7444, 959 .

Alves, M. T. G., \& Soares, J. F. (2008). O efeito das escolas no aprendizado dos alunos: um estudo com dados longitudinais no ensino fundamental. Educação e Pesquisa. São Paulo, 34(3), 527-544.

Amaral, L. F. L. E. do., \& Menezes-Filho, N. A. (2008). A relação entre gastos educacionais e desempenho escolar. Anais. Rio de Janeiro: ANPEC.

Amaral, L. F. L. E. do., \& Menezes-Filho, N. A. (2011). Os determinantes dos gastos educacionais e seus impactos sobre a qualidade do ensino. 2011. Dissertação (Mestrado em Economia) - Faculdade de Economia, Administração e Contabilidade, Universidade de São Paulo, São Paulo.

Angrist, J., \& Pischke, J.S. (2009).Most Harmless Econometrics: an empiricist's companion. Princeton, New Jersey: Princeton University Press.

Akaike, H. A new look at the statistical model identification. (1974). IEEE Transactions on Automatic Control. Boston, 19(6), 716-723.

Alon, S., \& Tienda, M. (2007). Diversity, opportunity and the shifting meritocracy in higher education. American Sociological Review. California, 72(4), 487511.

Arraes, R. A., \& Mariano, F.Z. (2019). Decomposição Quantílica Incondicional dos Diferenciais de Desempenho entre Alunos de Escolas Privadas e Públicas Profissionalizantes. Pesquisa e Planejamento Econômico (PPE). 49(3).

Backer, J. (2000). Evaluating the impact of development projects on poverty: a handbook for practitioners. Washington: The World Bank.

Barbetta, P. A., Andrade, D.F., \& Tavares, H.R. (2018). Estudos de Fatores Associados Através da Regressão Quantílica Hierárquica. Estudos em Avaliação Educacional29(71), 320-349.

Barros, R. P. de., \& Mendonça, R. (1996). O impacto do ambiente comunitário sobre o desempenho educacional. Rio de Janeiro: IPEA.

Becker, S., \& Ichino, A. (2002). Estimation of average treatment effects based on propensity scores. The Stata Journal, 2(4), 358-377.

Bittar, Eduardo C.B.; Almeida, Guilherme Assis de. (2005). Curso de Filosofia do Direito. 4. ed. São Paulo: Atlas. 
Research, Society and Development, v. 11, n. 1, e0111123948, 2022

(CC BY 4.0) | ISSN 2525-3409 | DOI: http://dx.doi.org/10.33448/rsd-v11i1.23948

Bonamino, Alicia Catalano de. (2002). Tempos de avaliação educacional: o SAEB, seus agentes, referências e tendências. Rio de Janeiro: Quartet.

Brandão Junior, J., \& Amaral, Sérgio Tibiriçá. (2007). Ações afirmativas: aspectos gerais. Etic-encontro de iniciação científica. Anais..., Presidente Prudente, $3(3)$.

BRASIL. Congresso Nacional. (2012). Lei $n^{\circ} 12.711$, de 29 de agosto de 2012. Dispõe sobre o ingresso nas universidades federais e nas instituições federais de ensino técnico de nível médio e dá outras providências. Brasília, DF.

BRASIL. Ministério da Educação. (2015). Entenda as cotas para quem estudou todo o ensino médio em escolas públicas. Brasília.

Cameron, A. C., \& Trivedi, P. K. (2009). Microeconometrics using stata. Texas: Stata press.

Cavalcanti, I. T. N., Andrade, C. S. M., Tiryaki, G. F., \& Costa, L. C. C. (2009). Desempenho acadêmico e o sistema de cotas no ensino superior: evidência empírica com dados da Universidade Federal da Bahia. Avaliação: Revista da Avaliação da Educação Superior. São Paulo. 24, 305-327

Farias, P. P. (2013). Os determinantes do desempenho acadêmico dos estudantes de ciências econômicas da UFC. 2013. Monografia de Graduação - Curso de Ciências Econômicas, Universidade Federal do Ceará, Fortaleza.

Fernandes, R., \& Gremaud, A. P.. (2009). Qualidade da educação: avaliação, indicadores e metas. In: Educação básica no Brasil: construindo o país do futuro.

Fonseca, J. S. Da., \& Martins, G. A. (1987). Curso de estatística. 3 ed. São Paulo: Atlas.

Fryer, R. G., \& Loury, G. C. (2005). Affirmative Action and its Mythology. Journal of Economic Perspectives, Washington D.C, 19(3), 147-162.

Freire, G. (1951). Sobrados e mucambos: decadência do patriarcado rural e desenvolvimento do urbano. 2. ed. ampl. Rio de Janeiro: José Olympio.

Furtado, C. (1954). A economia brasileira: contribuição a análise do seu desenvolvimento. Rio de Janeiro: Noite.

Gil, A. C. (2008). Métodos e técnicas de pesquisa social. São Paulo: Atlas.

Gimenes, N. (2015). Avaliação em larga escala no Brasil: tensões e desafios. Estudos em Avaliação Educacional. São Paulo, 26(62), 254-261.

Gomes, V. S. (2017). O impacto das políticas de cotas no ensino superior brasileiro: uma análise a partir do ENADE para os cursos de engenharia nos anos de 2008, 2011 e 2014. Trabalho de Conclusão de Curso (Graduação em Ciências Econômicas) - Universidade Federal de Uberlândia, Uberlândia.

Guarnieri, F. V., \& Melo-Silva, L. L. Ações afirmativas na educação. Psicol. Soc. 9(2), 70-78.

Holanda, S. B. De. (1956). Raízes do Brasil. 3. ed. Rio de Janeiro: José Olympio.

IBGE - Instituto brasileiro de geografia e estatistica. (2013). Estatísticas do cadastro central de empresas 2011. Rio de Janeiro: IBGE, 191.

INEP - Instituto nacional de estudos e pesquisas educacionais anísio teixeira. (2013). Censo da Educação Básica: 2012 - Resumo técnico. Brasília: INEP, 41.

INEP - Instituto nacional de estudos e pesquisas educacionais anísio teixeira. (2013). Manual do ENADE 2013.

Koenker, R., \& Bassett, G. Regression quantiles. (1978). Econometrica, New York, 46(1), 33-50.

Koenker, R., \& Hallock, K. (2000). Quantile Regression: An Introduction.

LDB - Leis de Diretrizes e Bases. Lei no 9.394. 1996.

Leon, F.L.L De.; Menezes-filho, N. A. (2003). Reprovação, avanço e evasão escolar no Brasil. Pesquisa e Planejamento Econômico. 32 (3), 417-452.

Libâneo, J. C. (1994). Didática.4 ed. São Paulo: Cortez.

Mendes, I. M. B. R., De Souza, W. P. S. F., Da Silva, A.C.A. (2020). Desigualdade de oportunidades e acesso à informação: evidências para a Universidade Federal da Paraíba. XXV Encontro Regional de Economia (ANPEC).

Menezes-Filho, N. A. (2001). Educação e desigualdade. In: LISBOA, M., MENEZES-FILHO, N. A. (orgs.). Microeconomia e sociedade no Brasil. Rio de Janeiro: EPGE.

Menezes-Filho, N. A.; Komatsu, B. K. (2019). Revisitando a Mobilidade Intergeracional de Educação no Brasil. Revista Brasileira De Economia. 73, 159180.

Moehlecke, S. (2002). Ação afirmativa: história e debates no Brasil. Cad. Pesqui. São Paulo, 117, 4.

Nascimento, A. Do. (2003). As políticas de ação afirmativa como instrumentos de universalização de direitos. Revista Lugar Comum - Estudos de Mídia, Cultura e Democracia. Rio de Janeiro, 18.

Paula, A. S. De. (2004). Ação afirmativa: instrumento de cidadania ou discriminação reversa? Semina: Ciências Sociais e Humanas, Londrina. Paraná, 25, 317.

Piscino, M. R. P. (2006). Teoria da ação afirmativa. 2006. Trabalho de Conclusão de Curso - UniFMU, São Paulo. 
Research, Society and Development, v. 11, n. 1, e0111123948, 2022

(CC BY 4.0) | ISSN 2525-3409 | DOI: http://dx.doi.org/10.33448/rsd-v11i1.23948

Pontes; L. A. F., \& Soares, T. M. (2016). As metas escolares do Ideb: uma proposta alternativa de cálculo. Estudos em Avaliação Educacional. São Paulo, 27(66), 690-715.

Rodrigues, L. O.: Ensaios sobre diferencial de desempenho escolar entre alunos de escolas rurais e urbanas no Brasil. 2017. Dissertação de mestrado da Universidade Federal do Ceará, centro de Ciências Agrárias, Programa de Pós-Graduação em Economia Rural, Fortaleza, 2017.

Rosenbaum, P., \& Rubin, R. (1983). The Central Role of the Propensity Score in Observational Studies for Causal Effects. Biometrika, 70(1), 41-55.

Sander, R. H. (2004). A systemic analysis of affirmative action in American law schools. Standford Law review. Stanford, 57(57), 367.

Santos, L. G. S. (2015). Ação afirmativa em universidades públicas brasileiras: diagnóstico da performance de beneficiados. 2015. Monografia (Bacharelado em Ciências Econômicas), Universidade de Brasília, Brasília.

Severino, A. J. (2013). Metodología do trabalho científico. São Paulo: Cortes.

Silva, A. F., Almeida, A. T. C., Lombardi Filho, S. C., \& Ramalho, H. M. B. (2019). Efeitos de Políticas Afirmativas sobre Esforço e Abandono: Evidências a partir da Universidade Federal da Paraíba. In: XXIV Encontro regional de economia, 2019, Fortaleza. Anais do XXIV Encontro Regional de Economia.

Sowell, T. (2004). Affirmative action around the world: an empirical study. New Haven: Yale University Press.

$\mathrm{Su}, \mathrm{X}$. (2005). Education hierarchy: within-group competition and affirmative action. SSRN, United States.

Tomei, M. (2005). Ação afirmativa para a igualdade racial: características, impactos e desafios. Brasília: OIT, 2005. Documento de trabalho elaborado no âmbito do Projeto Igualdade Racial OIT/Brasil.

Velloso, J. (2009). Cotistas e não-cotistas: rendimento de alunos da Universidade de Brasília. Cad. Pesqui., São Paulo, 39(137), 621-644.

Vilela, L., Tachibana, T. Y., Menezes-Filho, N., \& Komatsu, B. (2017). As cotas nas universidades públicas diminuem a qualidade dos ingressantes?. Estudos em Avaliação Educacional. São Paulo. 28, 652.

Zainko, M. A. S. (2008). Avaliação da educação superior no brasil: processo de construção histórica. Avaliação: Revista da Avaliação da Educação Superior. São Paulo. 13(3), 827-831. 